\title{
Multimodal Graph-Theoretic Approach for Segmentation of the Internal Limiting Membrane at the Optic Nerve Head
}

\author{
Mohammad Saleh Miri ${ }^{1,3}$, Victor A. Robles ${ }^{1,3}$, Michael D. Abràmoff ${ }^{2,1,3}$, \\ Young H. Kwon ${ }^{2}$, and Mona K. Garvin ${ }^{3,1}$ \\ ${ }^{1}$ Electrical and Computer Engineering, The University of Iowa, Iowa City, IA, USA \\ ${ }^{2}$ Ophthalmology and Visual Sciences, The University of Iowa, Iowa City, IA, USA \\ ${ }^{3}$ Iowa City VA Healthcare System, Iowa City, IA, USA
}

\begin{abstract}
In this work, we present a multimodal multiresolution graphbased method to segment the top surface of the retina called the internal limiting membrane (ILM) within optic-nerve-head-centered spectraldomain optical coherence tomography (SD-OCT) volumes. Having a precise ILM surface is crucial as this surface is utilized for measuring several structural parameters such as Bruch's membrane opening-minimum rim width (BMO-MRW) and cup volume. The proposed method addresses the common current segmentation errors due to the presence of retinal blood vessels, deep cupping, or a very steep slope of the ILM. In order to resolve these issues, the volume is resampled using a set of gradient vector flow (GVF) based columns. The GVF field is computed according to an initial surface segmentation which is obtained through a multiresolution framework. The retinal blood vessel information (obtained from corresponding registered fundus photographs) along with shape prior information are incorporated in a graph-theoretic approach to compute the ILM segmentation. The method is tested on the SD-OCT volumes from 44 glaucoma subjects and significantly smaller errors were obtained than that from current approaches.
\end{abstract}

\section{Introduction}

The internal limiting membrane (ILM) is the first intraretinal surface and is utilized for measuring several structural parameters such as Bruch's membrane opening-minimum rim width (BMO-MRW), total retinal thickness and cup volume. For example, the BMO-MRW, which is measured as the minimum Euclidean distance between the BMO and the ILM surface, is being recognized as a more sensitive metric for the diagnosis of glaucoma than traditional 2D metrics [1]. Furthermore, having a precise ILM surface segmentation is crucial as it plays an important role in extracting different features from SD-OCT volumes in approaches that utilize machine learning techniques for segmenting different retinal structures such as retinal blood vessels, optic disc, and optic cup [2-4].

However, precisely segmenting the ILM surface in optic nerve head (ONH)centered OCT volumes, as needed for computing parameters such as the BMOMRW of glaucoma patients, can be a challenging task. Since glaucoma patients

X. Chen, M. K. Garvin, J. Liu, E. Trucco, Y. Xu (Eds.): OMIA 2015, Held in Conjunction with MICCAI 2015, Munich, Germany, Iowa Research Online, pp. 57-64, 2015. Available from: http://ir.uiowa.edu/omia/2015_Proceedings/2015/ 
typically have deeper cups, in order to be able to follow the rapid changes in the shape of the ONH, steep slopes must be allowed in the ILM surface. Many current graph-based segmentation approaches [5,2] (often initially designed to segment the intraretinal layers of the macula or the peripapillary region surrounding the $\mathrm{ONH}$ ) cannot catch the deep, steeply sloped cups due to the fact that each A-scan corresponds to a column in the graph construction and the ILM surface is allowed to intersect with each column only once. In patients with larger and deeper cups, in order to be able to segment the ILM surface precisely, the segmentation must intersect with each A-scan multiple times (Fig. 1(a)). Therefore, the choice of method for building columns in the graph construction is important. The presence of large retinal blood vessels also makes a precise segmentation of the ILM at the ONH challenging as the blood vessels often block the underlying ILM and cause current segmentation approaches to include blood vessels and surrounding gaps as part of the ILM surface (Fig. 1(b)).

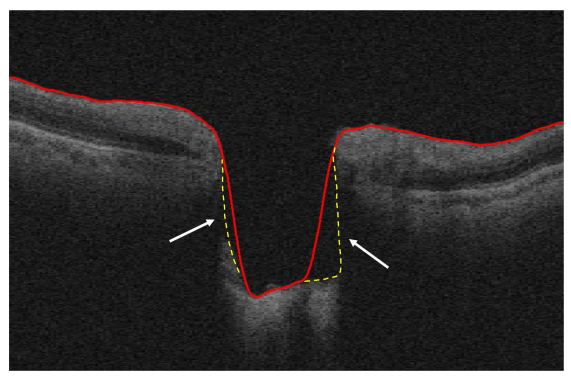

(a)

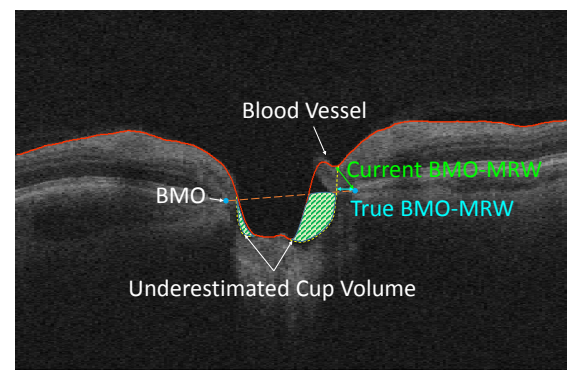

(b)

Fig. 1. Examples of ILM surface segmentation errors (shown with the arrows) of prior work. (a) Errors due to the steep slopes as well as low signal strength. (b) Errors due to the presence of blood vessels and resulting errors in computing cup volume and BMO-MRW due to the incorrect ILM segmentation. The orange dashed line is the BM reference surface, red lines indicate segmentation results using the approach of Lee et $a l$. [2] and the yellow dashed lines indicate the desired segmentation results.

In order to resolve these segmentation issues, we present a multimodal graphtheoretic approach that is capable of dealing with deep cupping as well as retinal blood vessels. To allow for steep and deep cupping, a new set of equally spaced columns along the normals of the ILM surface are utilized to resample the OCT volume. Since the columns in the graph construction must be non-overlapping (otherwise it may lead to a self-intersecting surface segmentation), we used the direction of the gradient vector flow (GVF) field $[6,7]$ to construct the new nonoverlapping columns. As opposed to the original ILM surface, the ILM surface in the resampled volume changes very smoothly, hence, we incorporate this prior shape information in the graph-theoretic approach [8]. The GVF-based columns are computed utilizing an initial ILM segmentation which is obtained through 
a multiscale process. Furthermore, in order to compensate for the effect of the blood vessels, the vessel mask from registered fundus photographs is used to correct the initial segmentation as well as modify the cost function that is used in the graph-based segmentation.

\section{Methods}

\subsection{Preprocessing and Initialization}

The flowchart of the proposed method is shown in Fig. 2. As part of the preprocessing, the corresponding fundus photographs are registered to the 2D SD-OCT projection image [2] using an ICP-based approach [9]. Because the retinal vessels inside the $\mathrm{ONH}$ are more visible in fundus photographs, the retinal blood vessels are segmented from the fundus photographs [10] and mapped to the SD-OCT volumes. Additionally, the SD-OCT volume $(x \times y \times z)$ is transferred to the radial domain $(r \times \theta \times z)$ with angular resolution of one degree (180 B-scans) to allow for a more consistent shape of the ILM across all slices.

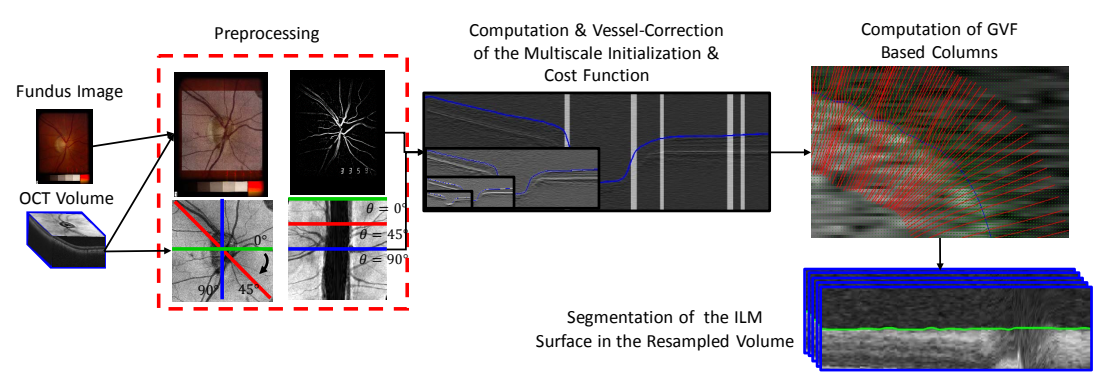

Fig. 2. Flowchart of proposed algorithm.

The initialization that is used for computing the gradient vector flow field is obtained through a multiresolution process. Since this step is only used for computing the GVF columns, here, we use a simple but reasonably accurate gradient-based peak detection method. The volumes are downsampled (by 8, 4, and 2) to three lower resolutions and a 3D Gaussian derivative filter identifies the intensity transitions from dark-to-bright (with high responses at the ILM surface). The response of the 3D Gaussian filter in the highest resolution, $\mathcal{E}(r, \theta, z)$, will be used as part of the cost function computation of the graph-theoretic approach in section 2.3. The peak detector looks for the first dominant peak from the top of each A-scan which belongs to the ILM surface. The searching interval in the next higher resolution at each A-scan is defined by the location of the peak in the previous lower resolution (the search interval for the lowest resolution includes the entire A-scan). The advantage of obtaining the initialization 
through a multiresolution process is that first, by downsampling the volumes, the speckle noise of the images is significantly reduced which helps the peak detector not be distracted by noise. Second, constraining the possible surface locations in subsequent resolutions helps avoid finding edges due to other surfaces.

\subsection{Gradient Vector Flow Computation}

In order to be able to follow the steep surfaces in cases of deep cupping, we use a set of equally spaced non-overlapping columns along the normals of the initial segmentation to resample the volume. The new columns are computed based on the direction of the GVF field and are suitable to be utilized for graph construction as GVF guarantees non-overlapping columns. Consider an SD-OCT volume in the radial domain $I(r, \theta, z)$. GVF is the vector field $\boldsymbol{V}(r, \theta, z)=$ $[u(r, \theta, z), v(r, \theta, z), w(r, \theta, z)]$ that minimizes the energy function $E[6]$ :

$$
E=\iiint \mu|\nabla \boldsymbol{V}|^{2}+|\nabla I|^{2}|\boldsymbol{V}-\nabla I|^{2} d r d \theta d z
$$

where $\mu$ is the regularization parameter. Due to the smooth shape of ONH, $\mu$ was not a sensitive parameter for computing GVF field and was set empirically to 0.02 . The vector field in Eq. 1 can be found by solving the following Euler equations:

$$
\begin{gathered}
\mu \nabla^{2} u-\left(u-I_{r}\right)|\nabla I|^{2}=0, \\
\mu \nabla^{2} v-\left(v-I_{\theta}\right)|\nabla I|^{2}=0, \\
\mu \nabla^{2} w-\left(w-I_{z}\right)|\nabla I|^{2}=0,
\end{gathered}
$$

where $\nabla I=\left(I_{r}, I_{\theta}, I_{z}\right)$ and $I_{r}, I_{\theta}$, and $I_{z}$ are the derivatives in $r, \theta$, and $z$ directions, respectively. Therefore, in order to compute the appropriate GVF field we need to have an initial vector field, $\nabla I$, with high gradient at the initial segmentation. Before computing the GVF field, in order to deal with the effect of blood vessels, the initial segmentation is corrected for the presence of retinal blood vessels by using $3 \mathrm{D}$ interpolation at the blood vessel locations. If $\mathcal{S}_{\text {initial }}(r, \theta)$ represents the blood-vessel-corrected initial segmentation, the initial vector field, $\nabla I$, is derived from the following $3 \mathrm{D}$ binary function $I(r, \theta, z)$ :

$$
I(r, \theta, z)=\left\{\begin{array}{ll}
0, & \mathcal{S}_{\text {initial }}>z \\
1, & \mathcal{S}_{\text {initial }}<z
\end{array} .\right.
$$

Starting from a point on the initial surface $\mathcal{S}_{\text {initial }}(r, \theta)$, the GVF-based columns are built by following the gradient flow. In order to find the coordinates of the next point on the column, the direction of the normalized gradient vectors in the neighborhood of the current point $\left\{\boldsymbol{V}_{i} /\left|\boldsymbol{V}_{i}\right| \mid i \in \mathcal{N}_{c}\right\}$, is interpolated to find the resultant direction $\boldsymbol{V}_{R}$. In order to avoid sampling artifacts, at each point, we take a small step $s$ (which must be smaller than half of the distance between two voxels in the volume) in the direction of the resultant vector. The columns are built by continuously moving in the direction of the gradient flow at both sides of the initial surface for the desired length of the columns (100 in this study) (Fig. 2). 


\subsection{Graph Construction and Cost Function Computation}

The GVF-based columns computed in section 2.2 are utilized to resample the OCT volume and they also serve as the columns in the graph construction. Since the GVF-based columns are along the normals of the initial segmentation, the ILM surface in the resampled volume is much smoother than the ILM surface before volume resampling. We benefit from this prior shape information to deal with the issue of presence of blood vessels. Here, we use the general graph-theoretic method proposed in $[5,8]$. Consider a volumetric image in the resampled volume described as $\mathcal{I}(i, j, k)$ with dimensions $I \times J \times K$, and the ILM surface $\mathcal{S}$ can be defined as a function $\mathcal{S}(i, j)$ that maps each $(i, j)$ pair to its corresponding $k$ value. The surface $\mathcal{S}$ intersects with only one voxel of each GVF-based column in the resampled domain, parallel to the $k$-axis and spans the entire $i \times j$ domain. The smoothness constraint represents the maximum distance allowed in $i$-direction $\Delta_{i}$ and in $j$-direction $\Delta_{j}$. In other words, if $\mathcal{I}\left(i, j, k_{1}\right)$ and $\mathcal{I}\left(i+1, j, k_{2}\right)$ are two adjacent voxels on the surface $\mathcal{S}$ in the $i$-direction then $\left|k_{1}-k_{2}\right| \leq \Delta_{i}$. Similarly, for two adjacent voxel on the surface $\mathcal{S}$ in the $j$-direction $\left(\mathcal{I}\left(i, j, k_{1}\right)\right.$ and $\left.\mathcal{I}\left(i, j+1, k_{2}\right)\right)$ we have $\left|k_{1}-k_{2}\right| \leq \Delta_{j}$.

In order to incorporate the shape prior information, in addition to hard smoothness constraints, the deviation from the expected shape inside the allowed constraints is penalized [8]. A convex function $f(h)$ penalizes the cost of the surface set if the change of the surface is deviated from the expected shape. Specifically, for any pair of neighboring columns (identified by $\left.\mathcal{N}_{c}\right) p=\left(i_{1}, j_{1}\right)$ and $q=\left(i_{2}, j_{2}\right)$ on surface $\mathcal{S}(i, j)$, if the expected shape change of surface $\mathcal{S}$ between $(p, q)$ is $m_{\left(i_{1}, j_{1}\right),\left(i_{2}, j_{2}\right)}$ the cost of the shape term can be written as:

$$
C_{\text {shape }}=\sum_{\left\{\left(i_{1}, j_{1}\right),\left(i_{2}, j_{2}\right) \in \mathcal{N}_{c}\right\}} f\left(\mathcal{S}\left(i_{1}, j_{1}\right)-\mathcal{S}\left(i_{2}, j_{2}\right)-m_{\left(i_{1}, j_{1}\right),\left(i_{2}, j_{2}\right)}\right) .
$$

Here, $\mathcal{N}_{c}$ indicates the neighboring relationship and $m_{\left(i_{1}, j_{1}\right),\left(i_{2}, j_{2}\right)}=0$ as since we resampled the volume along the normals of the initial segmentation, we expect to have very smooth ILM surface in the resampled volume. In addition, the penalizing convex function $f$ used here is a quadratic function.

As part of the total cost function of the ILM surface in the resampled volume, $C_{\mathcal{S}}$, we use an edge-based cost function called the on-surface cost function [5] which reflects the unlikelihood of a voxel being located on a specific surface. At the vessel locations, we want to rely more on the contextual and expected shape information as well as the feasibility constraints than on the on-surface cost function. Therefore, using the vessel information from segmented fundus photographs, we modify the cost function values at the blood vessel locations. Hence, the on-surface cost function can be expressed as:

$$
C_{\text {edge }}=\sum_{\{(i, j, k) \mid k=\mathcal{S}(i, j)\}} w(i, j) \dot{\mathcal{E}}(i, j, k),
$$

where $w(i, j)$ controls the modification of the cost function at the blood vessel locations. Here, we simply lower the weights of the blood vessel locations to be 
one-third of that of non-vessel locations. $\mathcal{E}(i, j, k)$ is computed by resampling the vessel-corrected inverted response of the $3 \mathrm{D}$ Gaussian derivative filter $\mathcal{E}(r, \theta, z)$ computed in section 2.1 using the GVF-based columns computed in section 2.2. The inversion is due to the fact that the on-surface cost function reflects the unlikelihood of a voxel being located on a specific surface; therefore, the intensities of $\mathcal{E}(r, \theta, z)$ must be normalized and inverted. The total cost of finding the ILM surface $\mathcal{S}$ in the resampled volume can be written as follows:

$$
C_{\mathcal{S}}=C_{\text {edge }}+\alpha C_{\text {shape }} .
$$

The coefficient $\alpha$ was set to 0.85 . The optimal surface can be found by computing the $\max -$ flow/min-cut in the arc-weighted graph as in [8]. In the end, the ILM segmentation is transferred back to the radial domain.

\section{Experiments and Results}

The dataset includes 44 optic nerve head-centered SD-OCT scans (from a $6 \mathrm{~mm}$ $\times 6 \mathrm{~mm} \times 2 \mathrm{~mm}$ region which contained $200 \times 200 \times 1024$ voxels) obtained from 44 patients with varying stages of glaucoma using a Cirrus (Carl Zeiss Meditec, Inc., Dublin, CA) SD-OCT scanner. As previously mentioned, each volume is resampled to the radial domain. The reference standard was obtained by randomly selecting two radial slices from each volume and performing the manual delineation of the ILM surface by an expert. The metrics used to gauge the accuracy of the segmentation results consisted of the signed and unsigned border positioning errors calculated in the radial domain. The unsigned border positioning error was calculated by averaging the distances between all surface points (on two randomly selected slices) from the reference standard and the corresponding closest points from the segmentation result. The signed border positioning error was similarly calculated but the signs of the distances were retained. If the algorithm's surface point was above the surface point of the reference standard, the sign was considered positive. Additionally, we compared the segmentation accuracies of the initialization used in the proposed method as well as the one proposed by Lee et al. [2].

Fig. 3 shows two example ILM segmentations on a single radial slice from the entire volume. The quantitative evaluations are provided in Table 3. The proposed method had significantly smaller signed and unsigned border positioning errors than the initialization and Lee et al. segmentations ( $p$-value $<0.05)$. The proposed method improved the average unsigned border positioning error of Lee et al. method and the initialization by $47.95 \%$ and $68.09 \%$, respectively. Since the volumetric ground truth wasn't available, a direct cup-volume comparison was not feasible; however, Fig. 3(b) shows a failure of the Lee et al. method in a case with deep cupping which results in underestimating the cup volume.

\section{Discussion and Conclusion}

We have presented a multimodal graph-theoretic approach for segmenting the internal limiting membrane in optic-nerve-head-centered OCT volumes of glau- 


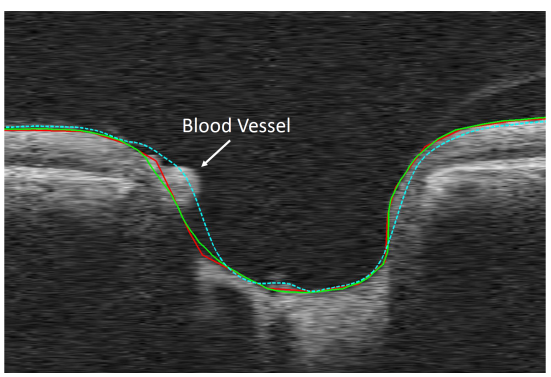

(a)

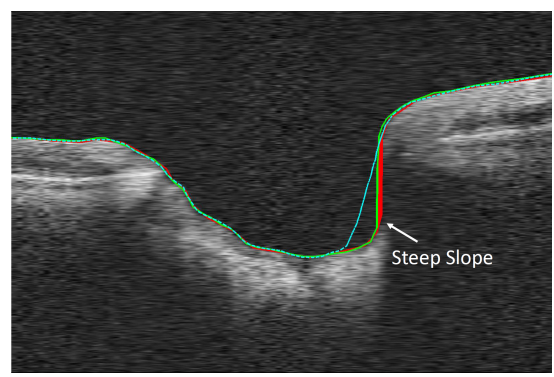

(b)

Fig. 3. Example results on the ONH portion of two slices from two volumes (only the ONH portion shown for better visibility). Red is the reference standard, green is the proposed algorithm and dashed cyan is Lee et al. [2] results. (a) Shows the effect of the presence of blood vessels and (b) shows an example of deep cupping.

Table 1. Average signed and unsigned border positioning error (Mean $\pm \mathrm{SD}$ in $\mu \mathrm{m}$ ).

\begin{tabular}{lcc}
\hline Methods & Unsigned & Signed \\
\hline Initialization & $20.12 \pm 10.36$ & $7.23 \pm 4.38$ \\
Lee et al. $[2]$ & $13.68 \pm 7.12$ & $5.21 \pm 3.45$ \\
Proposed method & $\mathbf{7 . 0 5} \pm \mathbf{3 . 4 3}$ & $\mathbf{- 2 . 0 7} \pm \mathbf{1 . 6 2}$ \\
\hline
\end{tabular}

coma patients. The proposed method addresses the segmentation errors of current approaches [2] due to the presence of the blood vessels and existing deep cupping and steep slopes in the ILM surface. The blood vessels were segmented from the corresponding registered fundus photographs and utilized to correct the multiscale initial segmentation and to modify the cost function used in the graph-theoretic approach at the vessel locations. The corrected initialization was then used in the computation of the non-overlapping GVF-based columns along the normals of the initial surface. The radial OCT volume was resampled using GVF-based columns such that the ILM surface converts to a very smooth surface. The expected shape of the ILM surface was incorporated in the total cost of the ILM surface respect to which the optimal surface was found using a graph-theoretic approach. While Lang et al. [11] showed the advantages of reformatting the macular OCT volume for purpose of intraretinal layer segmentation and GVF-based columns have been previously proposed for constructing the graph in other applications [7], this work presents a new framework for reformatting the OCT volumes using GVF-based columns in order to be able to segment the ILM at the ONH precisely. Furthermore, our use of a multimodal approach for the graph-construction and cost function design for use in the 3D graph-based approach that allows for incorporation of shape priors [8] is generally novel as well. 
Having a precise ILM segmentation is particularly important for computing modern glaucomatous parameters such as the BMO-MRW. However, in addition to allowing for a more precise ILM segmentation for ophthalmic applications, as in this work, it is expected that other application domains would benefit from a multimodal graph-construction and cost-function design approach.

Acknowledgements. This work was supported, in part, by the Department of Veterans Affairs Career Development Award 1IK2RX000728; the National Institutes of Health National Eye Institute R01 EY018853 and R01 EY023279; and the Marlene S. and Leonard A. Hadley Glaucoma Research Fund.

\section{References}

1. Chauhan, B.C., O'Leary, N., AlMobarak, F.A., Reis, A.S., Yang, H., Sharpe, G.P., Hutchison, D.M., Nicolela, M.T., Burgoyne, C.F.: Enhanced detection of open-angle glaucoma with an anatomically accurate optical coherence tomographyderived neuroretinal rim parameter. Ophth. 120(3) (2013) 535-543

2. Lee, K., Niemeijer, M., Garvin, M.K., Kwon, Y.H., Sonka, M., Abràmoff, M.D.: Segmentation of the optic disc in 3-D OCT scans of the optic nerve head. IEEE Trans. Med. Imag. 29(1) (2010) 159-168

3. Miri, M.S., Abràmoff, M.D., Lee, K., Niemeijer, M., Wang, J.K., Kwon, Y.H., Garvin, M.K.: Multimodal segmentation of optic disc and cup from SD-OCT and color fundus photographs using a machine-learning graph-based approach. IEEE Trans. Med. Imag. (2015)

4. Hu, Z., Niemeijer, M., Abràmoff, M.D., Lee, K., Garvin, M.K.: Automated segmentation of 3-D spectral OCT retinal blood vessels by neural canal opening false positive suppression. In: Proc. MICCAI 2010, LNCS. Volume 6363. (2010) 33-40

5. Garvin, M.K., Abràmoff, M.D., Wu, X., Russell, S.R., Burns, T.L., Sonka, M.: Automated 3-D intraretinal layer segmentation of macular spectral-domain optical coherence tomography images. IEEE Trans. Med. Imag. 28(9) (2009) 1436-1447

6. Xu, C., Prince, J.L.: Snakes, shapes, and gradient vector flow. IEEE Trans. Image Process. 7(3) (1998) 359-369

7. Oguz, I., Sonka, M.: LOGISMOS-B: Layered optimal graph image segmentation of multiple objects and surfaces for the brain. IEEE Trans. Med. Imag. 33(6) (2014) $1220-1235$

8. Song, Q., Bai, J., Garvin, M.K., Sonka, M., Buatti, J.M., Wu, X.: Optimal multiple surface segmentation with shape and context priors. IEEE Trans. Med. Imag. 32(2) (2013) 376-386

9. Niemeijer, M., Lee, K., Garvin, M.K., Abràmoff, M.D., Sonka, M.: Registration of 3 -D spectral OCT volumes combining ICP with a graph-based approach. In: Proc. SPIE, Med. Imag. 2012: Imag. Proc. Volume 8314. (2012) 83141A (9 pages)

10. Niemeijer, M., Staal, J., van Ginneken, B., Loog, M., Abràmoff, M.D.: Comparative study of retinal vessel segmentation methods on a new publicly available database. In: Proc. SPIE, Med. Imag. 2004: Imag. Proc. Volume 5370. (2004) 648-656

11. Lang, A., Carass, A., Calabresi, P.A., Ying, H.S., Prince, J.L.: An adaptive grid for graph-based segmentation in retinal OCT. In: Proc. SPIE, Med. Imag. 2014: Imag. Proc. Volume 9034. (2014) 903402 (9 pages) 